\section{Online Links}

Poliovirus: http://www.cdc.gov/doc. do/id/0900f3ec802286ba

VIRAL PATHOGENESIS

\title{
Cuckoos in an innate nest
}

Viruses are consummate cellular parasites, and it seems that for every strategy that the host deploys in the battle with its parasite, the virus responds by evading or subverting the host strategy in its quest for survival. Autophagy, a process that is used to degrade defunct cellular proteins and organelles in eukaryotes, can also degrade incoming pathogens as part of the host's innate immune armoury. However, reporting in PLoS Biology, Jackson et al. now reveal that some RNA viruses, including poliovirus, can subvert the autophagosome for viral replication and virus exit.

Upon infection of a eukaryotic cell with a positive-strand RNA virus, dramatic reorganization of cellular membranes takes place, which results in the formation of internal membrane structures that viruses use as a scaffold to replicate their RNA. Poliovirus infection induces the formation of 200-400 nm double-membrane vesicles, which accumulate in the cytoplasm and are associated with replicating viral RNA. Reasons

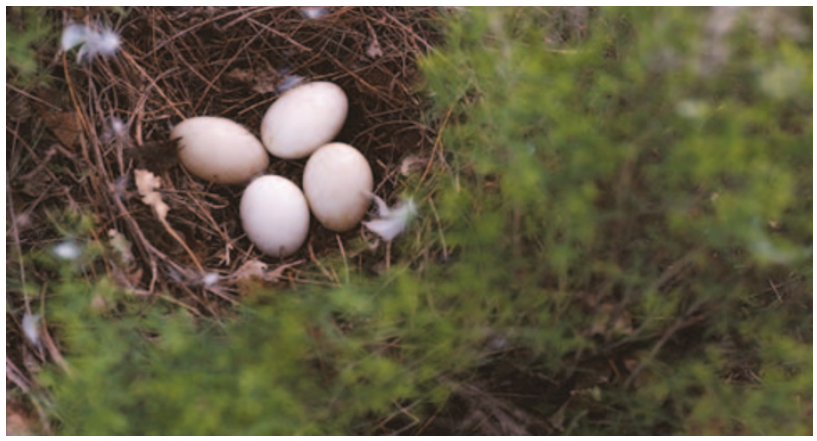

for the replication of viral genomic RNA on membranes might include facilitating the oligomerization and function of replicative proteins or enabling rapid replication before labile viral RNA is degraded.

Autophagy is thought to be conserved in yeast and humans. Autophagosomes either form de novo or are pinched off from the endoplasmic reticulum. Despite the paucity of proteins present in double-membrane autophagosomes, the authors showed that the virally induced membranes had several cell biological and immunomicroscopic hallmarks of bona fide autophagosomes. Perturbing the autophagosome pathway with drugs and RNA interference allowed them to determine whether the vesicles associated with poliovirus and rhinovirus infection might be autophagosomes.

Colocalization studies clearly showed that an autophagosomal marker, LC3, and a poliovirus replication protein were present on the same membranous structures within the cell. Furthermore, after poliovirus infection of cells, the cellular proteins LAMP1 and LC3 colocalized - this is usually only observed during autophagy. Treating cells with either poliovirus (or rhinovirus 2 and rhinovirus 14) or with drugs that induce autophagy resulted in the appearance of autophagosome-like double-membraned vesicles.

If autophagy is indeed co-opted by poliovirus to replicate its genome, it should be possible to perturb the autophagy process and affect the amount of virus produced in infected cells. Experiments using drugs that stimulated autophagy (tamoxifen and rapamycin) increased the intracellular yield of poliovirus in infected cells, whereas treatment with an autophagy inhibitor (3-methyladenine) reduced the amount of virus produced during infection. This link between autophagy and virus replication was probed further, using RNA interference to decrease the expression of autophagy proteins. Surprisingly, this not only decreased the levels of intracellular virions but drastically reduced the titre of extracellular viruses.

Therefore, as well as having a role in the replication, instead of destruction, of selected RNA viruses, it seems that autophagy might provide an exit route from the cell for newly replicated viruses in the absence of cell lysis. Understanding how RNA viruses replicate and exit from the cell, especially if this does not involve cell lysis - which might alert the adaptive and innate immune responses to counter virus infection - could be an important strategy by which viruses evade the host response.

Susan Jones

(1) References and links ORIGINAL RESEARCH PAPER Jackson, W.T. et al. Subversion of cellular autophagosomal machinery by RNA viruses. PloS Biol. 3, e156 (2005)

FURTHER READING Kirkegaard, K., Taylor, M. P. \& Jackson W. T. Cellular autophagy: surrender, avoidance and subversion by microorganisms. Nature Rev. Microbiol. 2, 301-314 (2004) 